\section{Atención odontológica de los niños en el marco de la pandemia del COVID-19}

\section{Children's dental care during the COVID-19 pandemic}

\section{Sr. Editor.}

Esta situación inusual que estamos viviendo, la pandemia del COVID-19 ${ }^{1,2}$ nos ha afectado a todos de alguna forma $\mathrm{y}$ en diferentes grados. Estamos pensando, evaluando y tratando de buscar una manera de sobrellevar esta situación. Han pasado más de 100 días de cuarentena obligatoria, durante dicho periodo se tuvo que dejar de atender a los pacientes de manera abrupta y de a poco estamos reiniciando las atenciones de manera cautelosa. La Odontopediatría no es ajena a esta situación, el especialista debe asumir el reto de atender a los niños con mayor precaución, cambiando muchos aspectos de la consulta. La prevención en Odontopediatría es muy importante, debido a que la evolución de los dientes marcará la salud bucodental del niño el resto de su vida. Nuestro deber es promover un buen estado de salud y enseñarles el cuidado de sus dientes y encías para que en el futuro no tengan problemas. Todo esto se logra realizando un trabajo conjunto con los padres con la finalidad que adquieran hábitos saludables. Se han desarrollado muchos documentos para el apoyo a los profesionales de la salud. La Asociación Latinoamericana de Odontopediatría (ALOP), ha emitido una serie de documentos y directivas para apoyar a la comunidad y a las familias en esta crisis sanitaria. Estas recomendaciones están basadas en la mejor evidencia disponible, tienen como objetivo brindar orientación básica a los padres cuyos hijos requieran tratamiento odontológico durante esta pandemia ${ }^{3}$. Se recomiendan procedimientos no invasivos, micro y mínimamente invasivos, los cuales tienen un enfoque conservador y biológico, eficacia comprobada y no requieren del uso de instrumentos rotatorios de alta velocidad o de jeringa triple generadoras de aerosoles. Estos tratamientos deben realizarse en dientes vitales que presenten desde lesiones iniciales de caries dental en esmalte, hasta cavidades con múltiples superficies comprometidas y sin signos ni síntomas de patología pulpar inflamatoria irreversible. Los pacientes deben ser incluidos en un programa de motivación y educación, que busque la desorganización de la biopelícula dental, por medio del cepillado dental y la adopción de una dieta baja en azúcares. Las radiografías intraorales ${ }^{4}$, deben realizarse solamente cuando sea absolutamente necesario para complementar al examen clínico, en lo posible debe emplearse la radiografía panorámica. Además, se recomienda una succión adecuada de la saliva y la atención a cuatro manos. Estas son las actividades clínicas sugeridas ${ }^{1-3}$ :

1. Cepillado Dental Supervisado, según protocolo establecido 5,6.

2. Barniz de flúor, según protocolo clínico establecido ${ }^{7,8}$.

\section{Carta al Editor}

\author{
Rosa Ana Melgar Hermoza 1,a \\ ${ }^{1}$ Universidad Peruana Cayetano Heredia, Facultad de \\ Estomatología, Lima, Perú. \\ a Doctora en Administración. Especialista en Odontope- \\ diatría.
}

\section{Correspondencia:}

Rosa Ana Melgar Hermoza: rosa.melgar@upch.pe Calle Gamma C-3, Urbanización Juan XXIII, San Borja, Lima, Perú

ORCID: 0000-0003-1124-9306

\section{Conflicto de intereses: ninguno.}


3. Fluoruro diamino de plata, según protocolo clínico establecido.

4. Resinas infiltrantes, según protocolo clínico establecido.

5. Sellantes de fosas y fisuras, según protocolo clínico establecido?.

6. Tratamiento restaurador atraumático, según protocolo clínico establecido ${ }^{10}$.

7. Remoción selectiva de tejido cariado, según protocolo clínico establecido ${ }^{10}$.

8. Manejo no restaurador de la cavidad, según protocolo clínico establecido ${ }^{8,10}$.

9. Técnica de Hall, según protocolo clínico establecido.

Indicaciones para la atención en Odontopediatría:

- El personal de apoyo debe estar preparado para realizar el protocolo de desinfección y toma de temperatura. Los padres deben conocer esto anticipadamente, para preparar al niño ya que estos cambios pueden afectar su percepción y crear ansiedad.

- La atención odontológica será, en un inicio, únicamente urgencias y emergencias, suspender el tratamiento dental no esencial o electivo hasta que nos encontremos en fases más avanzadas de la pandemia. Solo se atenderán: pulpitis irreversible severa, pericoronaritis, absceso dentoalveolar, alveolitis, hemorragia, traumatismo dentoalveolar con fractura dental, avulsión o luxación.

- El especialista debe estar atento a las necesidades y temores de los pacientes niños y sus padres. Además, realizar un adecuado manejo de conducta fomentando una comunicación acertiva de acuerdo a la edad de cada paciente.

- En lo posible realizar la atención mediante teleodontología, a través de un interrogatorio de los signos y síntomas del niño, que permitan realizar el diagnóstico. Usar fotos y videos, así como video llamadas, si el caso no se resuelve el paciente acudirá a la consulta.

- Informar a los padres y pacientes que la vestimenta del dentista ha cambiado, cubre más el cuerpo, puede incluir mameluco, batas o mandiles, además de la gorra, cubre zapato, respirador N95 o máscara quirúrgica y protección para los ojos con protectores laterales sólidos o un protector facial para proteger las mucosas, membranas de los ojos, nariz y boca durante los procedimientos que pueden generar gotículas de saliva, sangre u otros fluidos corporales, correspondientes al equipo de protección personal (EPP) ${ }^{2,3}$.

- En lo posible realizar tratamientos que no generen aerosoles.

- Las citas en el consultorios serán espaciadas, considerar los intervalos entre cada paciente, para la desinfección de los ambientes clínicos que podrían estar contaminadas, como sillones dentales, manijas, escupidera e instrumental ${ }^{1,2}$.

\section{Referencias bibliográficas}

1. Ather A, Patel B, Ruparel NB, et al. Coronavirus Disease 19 (COVID-19): Implications for clinical dental care. J Endod. 2020;46(5):584-95.

2. Ge ZY, Yang LM, Xia JJ, et al. Possible aerosol transmission of COVID-19 and special precautions in dentistry. J Zhejiang Univ Sci B. 2020;21(5):361-8. DOI: 10.1631/ jzus.B2010010

3. Meng L, Hua F, Bian Z. Coronavirus Disease 2019 (COVID-19): Emerging and Future Challenges for Dental and Oral Medicine. J Dent Res. 2020;99:481-7.

4. AAPD. Prescribing dental radiographs for infants, children, adolescents, and individuals with special health care needs. Pediatr Dent. 2017;39:205-7.

5. Walsh T, Worthington HV, Glenny AM, et al. Fluoride toothpastes of different concentrations for preventing dental caries. Cochrane Database Syst Rev 2019;3:Cd007868.

6. Dos Santos APP, de Oliveira BH, Nadanovsky P. A systematic review of the effects of supervised toothbrushing on caries incidence in children and adolescents. Int J Paediatr Dent. 2018;28:3-11.

7. Fluoride Therapy. Pediatr Dent. 2017;39(6):242-245.

8. Marinho VC, Worthington HV, Walsh T, et al. Fluoride varnishes for preventing dental caries in children and adolescents. Cochrane Database Syst Rev. 2013:Cd002279.

9. Slayton RL, Urquhart O, Araujo MWB, et al. Evidence-based clinical practice guideline on nonrestorative treatments for carious lesions: A report from the American Dental Association. J Am Dent Assoc. 2018;149:837-49.e19.

10. Li F, Jiang P, Yu F, et al. Comparison between Fissure Sealant and Fluoride Varnish on Caries Prevention for First Permanent Molars: a Systematic Review and Meta-analysis. Sci Rep. 2020;10(1):2578. DOI: 10.1038/ s41598-020-59564-5. 